\title{
DISCRETE QUADRATIC SPLINES
}

\section{SURENDRA SINGH RANA}

\author{
Department of Mathematics and Computer Science \\ R.D. University \\ Jabalpur 482001 \\ India \\ (Received July 22, 1987)
}

\begin{abstract}
In the present paper, we define a new class of discrete splines and study the existence, uniqueness, and convergence properties of discrete quadratic splines satisfying the Mean Averaging Condition.
\end{abstract}

KEY WORDS AND PHRASES. Discrete quadratic spline, existence, uniqueness and convergence, mean averaging condition. 1980 AMS SUBJECT CLASSIFICATION CODE. Primary 41A05, 65 D07.

1. INTRODUCTION.

Schoenberg [1] and DeBoor [2] have studied even degree splines whose integral means between the mesh points agree with the same means of a given function. Considering a more general condition viz the mean averaging condition (MAC), Sharma and Tzimbalario [3] have studied the existence, uniqueness and convergence of a quadratic spline satisfying MAC. It is of some interest to mention that Varga [4] has considered error bounds for splines satisfying conditions involving functionals using a different approach. The existence, uniqueness and convergence properties of discrete cubic splines satisfying MAC have been studied by Dikshit and Power [5]. It may be observed that the approach used by Lyche [6] for defining discrete cubic splines is not capable of providing the corresponding definition for discrete splines of degree less than three. In the present paper, we consider certain interpolatory discrete splines of degree two and study the existence, uniqueness and convergence properties of such splines satisfying MAC.

2. EXISTENCE AND UNIQUENESS.

Let $a$ mesh on $[a, b]$ be defined by $P: a=x_{0}<x_{1}<\ldots<x_{n}=b$ with $x_{1}-x_{1-1}=p_{1}$ for $1=1,2, \ldots, n$ and $p=\max _{1} p_{1}$. The class $D(2, p, h)$ of discrete quadratic splines for $h>0$ is the set of all continuous functions $s(x, h)$ such that for $1=1,2, \ldots, n$ the restriction $s_{1}$ of $s(x, h)$ on $\left[x_{1-1}, x_{i}\right]$ is a polynomial of degree 2 or less and 


$$
D_{h}^{\{1\}} s_{i}\left(x_{i}-h\right)=D_{h}^{\{1\}} s_{i+1}\left(x_{i}+h\right) ; i=1,2, \ldots, n-1
$$

where the central difference operator $D_{h}^{\{1\}} f(x)=(f(x+h)-f(x-h)) / 2 h$ (see Lyche [7]).

For convenience, we set for each $i$,

$$
\begin{aligned}
& A_{1}(j, k)=\int_{x_{i-1}}^{x_{i}}\left(x-x_{i-1}-h\right)^{j}\left(x_{1}-x-h\right)^{k} d g, j, k=0,1,2 \\
& H_{i} \cdot \int_{x_{i-1}}^{x_{i}} d g, F_{1}=\int_{x_{1-1}}^{x_{1}} f d g \text { and } J_{1}=\left[x_{1-1}+2 h, x_{1}-2 h\right] .
\end{aligned}
$$

In the present section, we shall study the following:

PROBLEM 2.1. Let $f$ be a b-a periodic locally integrable function with respect to nonnegative measure $\mathrm{dg}$. We investigate restrictions on $g$ such that there exists a unique $b-a$ periodic discrete quadratic spline $s(x, h)$ in $D(2, P, h)$ satisfying the MAC:

$$
\int_{x_{1-1}}^{x_{1}}(f(x)-s(x, h)) d g=0,1=1,2, \ldots, n .
$$

We shall answer the Problem 2.1 in the following,

THEOREM 2.1. Suppose that the support of the restriction of $g$ over $\left[x_{1-1}, x_{1}\right]$ is included in $J_{i}$ and $i$ ts total variation over $J_{1}$ is positive. Then there exists a unique discrete periodic quadratic spline $s(x, h)$ in $D(2, P, h)$ satisfying MAC (2.2).

REMARK. 2.1. In the case in which $h \rightarrow 0$, Theorem 2.1 gives the corresponding result for continuous quadratic spline interpolation under MAC studied in [3]. It is also interesting to note that condition (2.2) reduces to some other interpolating conditions by suitable choice of $g(x)$.

PROOF OF THEOREM 2.1. It is clear that $D_{h}^{\{1\}} s(x, h)$ is linear, hence in the interval $\left[x_{1-1}, x_{1}\right]$, we may write

$$
\left(p_{1}-2 h\right) D_{h}^{\{1\}} s(x, h)=\left(x-x_{1-1}-h\right) M_{1}+\left(x_{1}-x-h\right) M_{1-1}
$$

where $M_{i}=M_{1}(h)=D_{h}^{\{1\}} s\left(x_{1}-h, h\right)$. Thus, summing (2.3), we have

$$
2\left(p_{1}-2 h\right) s(x, h)=\left(x-x_{i-1}-h\right)^{2} M_{1}-\left(x_{1}-x-h\right)^{2} M_{1-1}+2\left(p_{1}-2 h\right) c_{1}
$$

where $c_{1}$ is a constant which has to be determined. Using the continuity of $s(x, h)$, we get

$$
\begin{aligned}
& 2\left(p_{i+1}-2 h\right)\left[\left(p_{1}-h\right)^{2} M_{1}-h^{2} M_{i-1}+2\left(p_{1}-2 h\right) c_{1}\right] \\
& =2\left(p_{1}-2 h\right)\left[h^{2} M_{1+1}-\left(p_{1+1}-h\right)^{2} M_{1}+2\left(p_{i+1}-2 h\right) c_{1+1}\right] .
\end{aligned}
$$


Since $s(x, h)$ satisfies the interpolatory condition (2.2), we get from (2.4)

$$
2\left(p_{1}-2 h\right) F_{1}=M_{1} A_{1}(2,0)-M_{1-1} A_{1}(0,2)+2\left(p_{1}-2 h\right) c_{1} H_{1}
$$

Eliminating $c_{1}, c_{1+1}$ between the equations $(2.5)-(2.6)$, we get the following system of equations:

$$
\begin{aligned}
\left(p_{1}-2 h\right) & {\left[d_{i+1}(2,0)-h^{2}\right] M_{1+1}+\left[\left(p_{i+1}-2 h\right)\left\{\left(p_{i}-h\right)^{2}-d_{1}(2,0)\right\}\right.} \\
& \left.+\left(p_{1}-2 h\right)\left\{\left(p_{1+1}-h\right)^{2}-d_{1+1}(0,2)\right\}\right] M_{1}+\left(p_{1+1}-2 h\right)\left[d_{i}(0,2)-h^{2}\right] M_{1-1} \\
& =2 F_{i}^{*}(h) .
\end{aligned}
$$

where

$$
\begin{aligned}
& F_{i}^{*}(h)=\left(p_{1}-2 h\right)\left(p_{1+1}-2 h\right)\left[F_{i+1} / H_{1+1}-F_{i} / H_{1}\right] \text { and } \\
& d_{i}(p, q)=A_{1}(p, q) / H_{1} \text { for } p, q=0,1,2, ; 1=1,2, \ldots, n .
\end{aligned}
$$

In order to prove Theorem 2.1, we shall show that the system of equations (2.7) has a unique set of solutions. Since the support of the restriction of $g$ over $\left[x_{1-1}, x_{1}\right]$ is included in $J_{1}$, we observe that the coefficients of $M_{1-1}, M_{1}$ and $M_{1+1}$ are all nonnegative by virtue of the hypothesis that the total variation of $g$ over $J_{i}$ is positive. Further, the excess of the positive value of the coefficient of $M_{1}$ over the sum of the positive values of the coefficients of $M_{1-1}$ and $M_{1+1}$ is

$$
t_{1}(h)=2\left[\left(p_{i}-2 h\right) R_{1+1}+\left(p_{1+1}-2 h\right) R_{1}\right]
$$

where $R_{1}=d_{1}(1,1)$ with $h=0$. Clearly $t_{1}(h)>0$ under the conditions of Theorem 2.1. Thus, the coefficient matrix of the system of equations (2.7) is diagonally dominant and hence invertible. This completes the proof of Theorem 2.1.

\section{ANOTHER INTERPOLATION PROBLEM.}

In this section of the paper, we shall answer problem 2.1 with MAC replaced by the interpolatory condition:

$$
s\left(x_{1-1}\right)+s\left(x_{1}\right)=f\left(x_{1-1}\right)+f\left(x_{1}\right), 1=1,2, \ldots \ldots, n .
$$

It may be mentioned that it is possible to deduce the interpolatory condition (3.1) from the MAC (2.2) by a suitable choice of dg. Further, it is easy to see that the conditions of Theorem 2.1 are not satisfied for the interpolatory condition (3.1). To answer Problem 2.1 for the interpolatory condition (3.1), we use (3.1) in (2.4) to get 


$$
2\left(p_{1}-2 h\right)\left(f_{1-1}+f_{1}\right)=\left(p_{1}^{2}+2 h^{2}-2 p_{1} h\right)\left(M_{1}-M_{1-1}\right)+4 c_{1}\left(p_{1}-2 h\right)
$$

where $f_{1}=f\left(x_{1}\right)$ for all $i$. Eliminating $c_{1}, c_{1+1}$ from the equations (3.2) and (2.5), we have the following system of equations:

$$
p_{1} M_{1-1}+\left(p_{1}+p_{1+1}\right) M_{1}+p_{1+1} M_{1+1}=2\left(f_{1+1}-f_{1-1}\right) \text {. }
$$

Clearly the system of equations (3.3) is not diagonally dominant. However, we may rewrite (3.3) in the following form,

$$
\begin{aligned}
& p_{1}\left(M_{1-1}+M_{1}\right)-2\left(f_{1}-f_{1-1}\right)+p_{1+1}\left(M_{1}+M_{1+1}\right)-2\left(f_{1+1}-f_{1}\right)=0 \\
& 1=1,2, \ldots, n .
\end{aligned}
$$

Assuming that $n$ is odd and $s, f$ are $b-a$ periodic, we get from (3.4) (cf. Sharma and Tzimbalario [3])

$$
M_{i}=\sum_{j=0}^{n-1}(-1)^{j} \frac{f_{i+j+1}-f_{i+j}}{P_{i+j+1}}
$$

Thus, we have proved the following:

THEOREM 3.1. For every b-a periodic function $f$, there exists a unique discrete periodic quadratic spline $s(x, h)$ in $D(2, P, h)$ satisfying the interpolatory condition (3.1) if and only if $\mathrm{n}$ is odd.

\section{ERROR BOUNDS.}

In this section, we shall estimate the error function $e(x)=s(x, h)-f(x)$ for the spline interpolant of Theorem 2.1. over the discrete interval $[a, b]_{h}$ which 18 the intersection of $[a, b]$ with

$$
R_{h a}=\{a+j h: j \text { is an integer, a is real }\} \text {. }
$$

For a function $f$ defined over $[a, b]_{h}, w(f, p)$ denotes the discrete modulus of continuity. Throughout this section for convenience, we assume that $P$ is a uniform mesh so that $x_{1}-x_{1-1}=p$ and

$$
g(x+p)-g(x)=K \text { (a constant). }
$$

We first begin with the following result, (see Lyche [7])

LEMMA 4.1. If $\left\{x_{i}\right\}_{i=0}^{l},\left\{\alpha_{1}\right\}_{i=0}^{i} \subset[a, b]_{h}$ for some $h>0$ and

$\left\{a_{i}\right\}_{i=0}^{n}, \quad\left\{b_{i}\right\}_{i=0}^{m}$ are given sequences of nonnegative real numbers such that

$\sum_{i=0}^{n} a_{1}=\sum_{i=0}^{m} b_{1}$, then for any real valued function $f$ defined on the discrete interval $[a, b]_{h}$, we have

$$
\left|\sum_{i=0}^{n} a_{1}\left[x_{0}, x_{1}\right] f-\sum_{i=0}^{m} b_{1}\left[\alpha_{0}, \alpha_{1}\right] f\right|<W\left(b_{h}^{\{1\}} f, b-a-h\right) \sum a_{1} \text {. }
$$


We now proceed to estimate the error bounds at the points $x_{1}$ h for all 1 . Observing that, $A_{i}(j, k)=A(j, k)$ and $H_{1}=H$ for uniform mesh, we shall prove the following,

LEMMA 4.2. Let $s(x, h)$ be the interpolating spline of Theorem 2.1. If $g$ is a step function with jumps of 1 only at the points of $[a, b]_{h}$, then

$$
\left\|\left(D_{h}^{\{1\}} e\left(x_{1}-h\right)\right)\right\|<p t(h) J_{1}(A, h) w\left(D_{h}^{\{1\}} f, p\right)
$$

where $t(h)$ is some positive function of $h$ and $J_{1}(A, h)$ is some positive function of $A(j, k)$ and $h$.

PROOF OF LEMMA 4.2. It may be observed that the system of equations (2.7) may be written as

$$
A(h) M(h)=2 F^{*}(h)
$$

where $A(h)$ is the coefficient matrix and $M(h)$ and $F(h)$ are single column matrices $\left(M_{i}(h)\right)$ and $\left(F_{i}^{*}(h)\right)$ respectively. Further, it may be seen that the row max norm of $A^{-1}(h)$ is

$$
\left\|A^{-1}(h)\right\|<t(h)
$$

where $t(h)=\max _{1}\left\{t_{1}^{-1}(h)\right\}$. From (4.3), it follows that

$$
A(h)\left(D_{h}^{\{1\}} e\left(x_{1}-h\right)\right)=2 F_{1}^{*}(h)-A(h) D_{h}^{\{1\}} f\left(x_{i}-h\right) \text {. }
$$

Using the fact that $g$ is a step function with jumps of 1 at the points of $[a, b]_{h}$, we wish to apply Lemma 4.1 to estimate the right hand side of (4.5). For this, we notice that $D_{h}^{\{1\}} f\left(x_{i}-h\right)=\left[x_{1}-2 h, x_{1}\right] f$ and that the sum of the coefficients of the $D_{h}^{\{1\}} f\left(x_{1}-h\right)$ 's on the right hand side of $(4.5)$ is

$$
\left[-2 p(p-2 h)^{2} / H\right] \int_{x_{1}}^{x_{i+1}} d g
$$

whereas

$$
2 F_{i}^{*}(h)=\left[-2 p(p-2 h)^{2} / H\right] \int_{x_{1}}^{x_{i+1}}[x, x+p] f d g .
$$

Thus, rearranging the terms suitably and then applying Lemma 4.1, we see that the row max norm of the matrix on the right hand side of (4.5) does not exceed

$$
p J_{1}(A, h) W\left(D_{h}^{\{1\}} f, p\right)
$$

where $J_{1}(A, h)$ is some positive function of $A(j, k)$ and $h .(4.2)$ now follows when we appeal to $(4.4)$. We are now set to prove the following: 
THEOREM 4.1. Let $s(x, h)$ be the interpolating spline of Theorem 2.1. If $g$ is a step function with jumps of 1 only at the points of $[a, b]_{h}$, then

$$
\| D_{h}^{\{1\}} e(x, h)||<J(A, h) W\left(D_{h}^{\{1\}} f, p\right)
$$

where $J(A, h)$ is some positive function of $A(j, k)$ and $h$.

PROOF OF THEOREM 4.1. In order to prove Theorem 4.1, we respectively replace $M_{i}(h)$ and $D_{h}^{\{1\}} s(x, h)$ by $D_{h}^{\{1\}} e\left(x_{1}-h\right)$ and $D_{h}^{\{1\}} e(x)$ in equation $(2.3)$. Now adjusting suitably the additional terms and using Lemma 4.1 , we see that

$$
\left\|D_{h}^{\{1\}} e(x)\right\|<\left\|\left(D_{h}^{\{1\}} e\left(x_{1}-h\right)\right)\right\|+w\left(D_{h}^{\{1\}} f, p\right) .
$$

Combining the estimate (4.2) with (4.7), we complete the proof of Theorem 4.1 with $J(A, h)=\left[1+p t(h) J_{1}(A, h)\right]$.

ACKNOWLEDGEMENTS. The author would like to express his sincere thanks to Professor H.P. Dikshit for some helpful discussions.

\section{REFERENCES}

1. SCHOENBERG, I.J., "Splines and Histograms" Splines and Approximation Theory, ISNM, 21, 277-327, Birkhäuser-Verlag, Bese1, 1973.

2. DE BOOR, C., Appendix to Splines and Histograms, ISNM 21, 329-358, Birkhauser-Verlag, Besel, 1973.

3. SHARMA, A. and TZIMBALARIO, J., Quadratic Splines, Jour. Approx. Theory, 19 (1977), 186-193.

4. VARGA, R.S., Error Bounds for Spline Interpolation in Approximation with Special Emphasis on Spline Functions (I.J. Schoenberg, Ed.), 367-388, Academic Press, New York, 1969.

5. DIKSHIT, H.P. and POWER, P., Area Matching Interpolation by Discrete Cubic Splines, Approximation Theory and Applications Proc. Int. Conf. St. John's New Foundland, (1984), 35-45.

6. LYCHE, T., Discrete Cubic Spline Interpolation, BIT, 16(1976), 281-290.

7. LYCHE, T., Discrete Cubic Spline Interpolation, Report, RRI5, University of 0s10, 1975. 


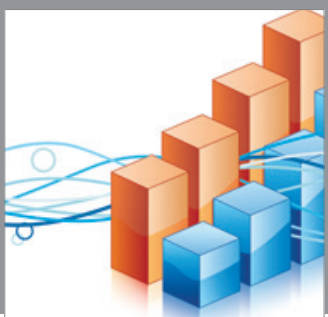

Advances in

Operations Research

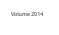

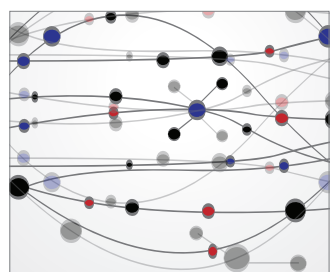

\section{The Scientific} World Journal
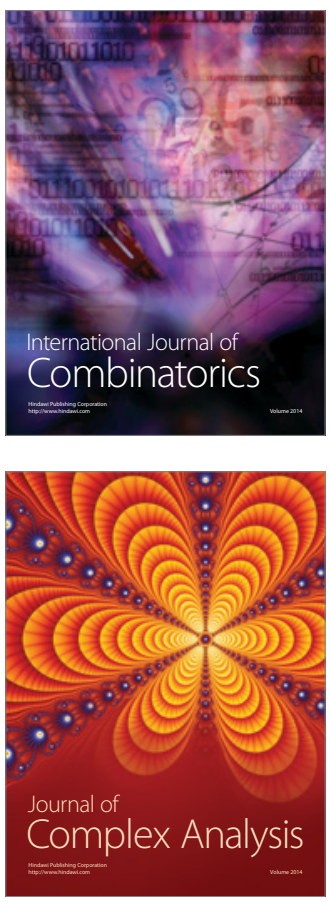

International Journal of

Mathematics and

Mathematical

Sciences
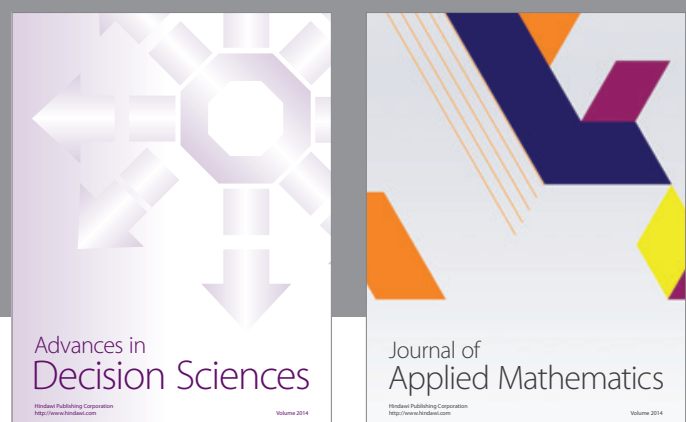

Journal of

Applied Mathematics
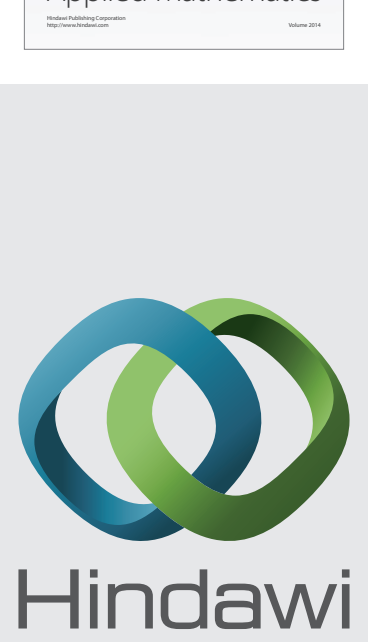

Submit your manuscripts at http://www.hindawi.com
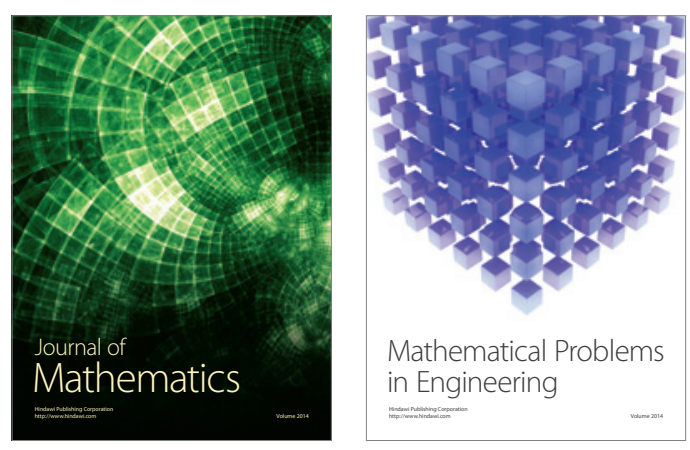

Mathematical Problems in Engineering
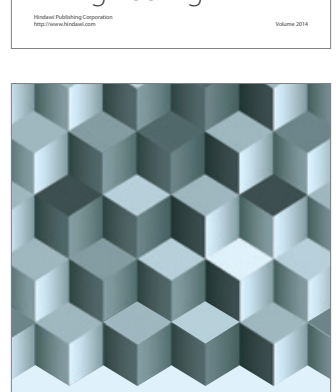

Journal of

Function Spaces
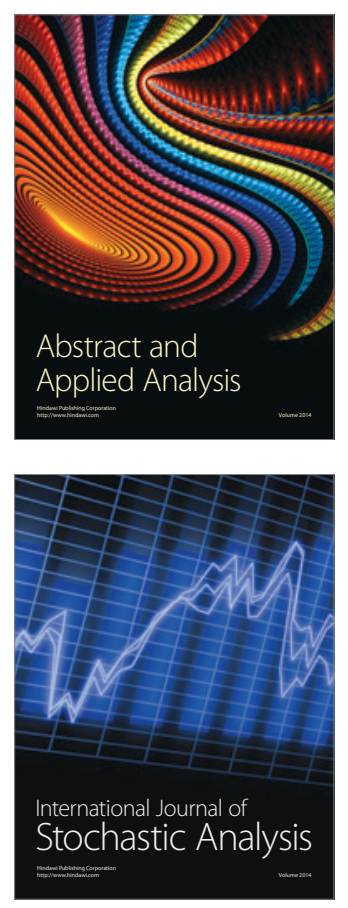

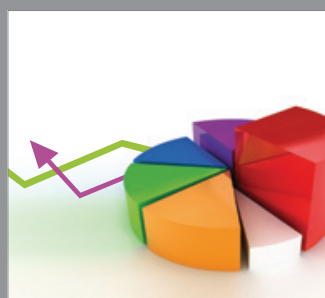

ournal of

Probability and Statistics

Promensencen
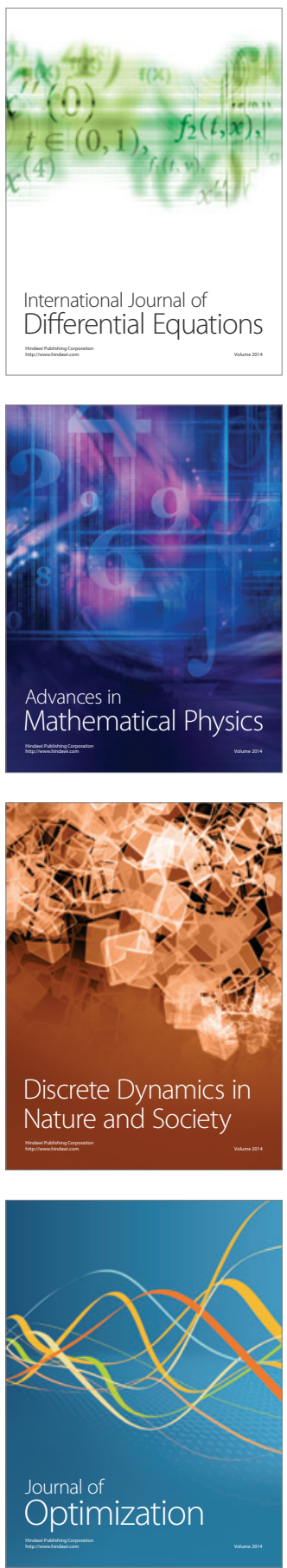